\title{
ARTICLE \\ Acid sphingomyelinase downregulation alleviates vascular endothelial leptin resistance in rats
}

\author{
Bei-bo Cai ${ }^{1}$, Yi-ni $L u^{1}$ and Ming $\mathrm{Xu}^{1}$
}

\begin{abstract}
Leptin resistance in endothelial cells leads to vascular endothelial dysfunction, which is the beginning and crucial link of atherosclerosis. However, the mechanism of leptin resistance remains obscure. Acid sphingomyelinase (ASM) catalyzes the hydrolysis of sphingomyelin to produce ceramide, which plays an important role in the progression of metabolic and cardiovascular diseases. In this study, we investigated whether ASM could regulate leptin resistance in vascular endothelial cells. We induced endothelial leptin resistance in rat aortic endothelial cells through treatment with palmitic acid ( $0.3 \mathrm{mM})$ or knockdown of leptin receptor $(\mathrm{Ob}-\mathrm{Rb})$, which resulted in the increase of suppressor of cytokine signaling 3 expression, the decrease of Ob-Rb expression, and signal transducer and activator of transcription 3 (STAT3) phosphorylation at Tyr705. We found that these indicators of leptin resistance were reversed by knockdown of ASM or by the selective ASM inhibitors amitriptyline (AMI) and imipramine (IMI). Supplementation of ceramide inhibited Ob-Rb expression and STAT3 phosphorylation by inhibiting extracellular signal-regulated kinase $1 / 2$ activation. Furthermore, we found that knockdown of ASM enhanced endothelial nitric oxide (NO) synthase activity and NO production, as well as the Akt phosphorylation at ser473, which was regulated by STAT3. High-fat diet (HFD) feeding-induced leptin resistance in rats in vivo; administration of $\mathrm{AMI}$ and IMI $\left(10 \mathrm{mg} \cdot \mathrm{kg}^{-1}\right.$ per day, intraperitoneally, for 2 weeks) increased the release of endothelial NO to relieve the vasodilatory response and improved the endothelial leptin resistance in the aorta of HFDfed rats. These results suggest that ASM downregulation reverses endothelial leptin resistance, and consequently improves vascular endothelial dysfunction. This study highlighted ASM as a potential therapeutic target for endothelial leptin resistance.
\end{abstract}

Keywords: leptin resistance; rat aortic endothelial cells; acid sphingomyelinase; ceramide; Ob-Rb; SOCS3; STAT3; ERK1/2; Akt; amitriptyline; imipramine; atherosclerosis

Acta Pharmacologica Sinica (2020) 41:650-660; https://doi.org/10.1038/s41401-019-0328-3

\section{INTRODUCTION}

Leptin is a hormone primarily secreted by adipocytes that regulates energy homeostasis [1]. In addition to having central effects in the brain, leptin has been found to exert a large number of peripheral actions in recent years. Leptin receptors broadly exist in the vascular endothelium, and intact leptin signaling in endothelial cells plays an important role in endothelial function [2]. Elevated leptin levels that lead to feeding disorders and energy imbalance define a state of the so-called leptin resistance [3]. Ob-Rb initiates leptin signaling through more than seven different signal transduction pathways, and excessive suppressor of cytokine signaling 3 (SOCS3) activity is a potential mechanism of leptin resistance [4]. Leptin resistance can lead to endothelial dysfunction, which represents the beginning of and is crucial link to atherosclerosis. To date, the mechanism of endothelial leptin resistance remains poorly understood. A more complete understanding of endothelial leptin resistance is necessary to develop effective therapeutics for atherosclerotic cardiovascular diseases.

Acid sphingomyelinase (ASM) is a water-soluble, lysosomal glycoprotein that catalyzes the degradation of membrane-bound sphingomyelin into phosphocholine and ceramide. ASM has two distinct forms: classic lysosomal ASM is independent of exogenous zinc and is critical for the catabolism of membrane sphingomyelin during the physiological turnover of membranes, and secretory ASM in secretory lysosomes generates ceramide at the outer plasma membrane to initiate transmembrane signal transduction [5]. Mutations in the gene encoding ASM (SMPD1) results in ASM deficiency, which causes Niemann-Pick disease types A and B [6]. ASM is highly expressed in endothelial cells [7], and some studies have found that ASM/ceramide signaling is associated with multiple diseases, such as depression and atherosclerosis [8, 9]. Our previous study found that ASM contributes to the formation of membrane raft/redox signalosomes and mediates redox signaling in coronary arterial endothelial cells [10]. Moreover, the inhibition of ASM can alleviate insulin resistance in endothelial cells of diabetic rats [11]. However, it has never been reported whether ASM can influence leptin resistance in vascular endothelial cells.

The present study investigated whether ASM downregulation can attenuate leptin resistance to alleviate vascular lesions and dysfunction. Here, ASM gene silencing and the selective inhibitors amitriptyline (AMI) and imipramine (IMI) were used to downregulate ASM. It has been reported that elevated saturated fatty concentrations induce leptin resistance and impair endothelial cell function [12]. Thus, we used $0.3 \mathrm{mM}$ palmitic acid (PA), a 16carbon saturated fatty acid, to induce leptin resistance in rat aortic

${ }^{1}$ Department of Clinical Pharmacy, School of Preclinical Medicine and Clinical Pharmacy, China Pharmaceutical University, Nanjing 210009, China Correspondence: Ming Xu (mingxu@cpu.edu.cn)

Received: 19 June 2019 Accepted: 31 October 2019

Published online: 17 December 2019 
endothelial cells (RAECs). In addition, Ob-Rb gene manipulation with small interfering RNA (siRNA) was used to produce another model of leptin resistance. Obesity represents a state of endothelial leptin resistance, so leptin resistance was generated by feeding rats a high-fat diet (HFD). Leptin also improved vascular endothelial function by activating the endothelial nitric oxide synthase (eNOS)/NO signaling pathway; however, this effect was impaired under leptin-resistant conditions. Therefore, the eNOS/NO pathway and relevant vascular functions were detected to further clarify the regulation of endothelial leptin resistance by ASM.

\section{MATERIALS AND METHODS}

Cell culture and reagents

RAECs were purchased from ATCC (The Global Bioresource Center, purchased from Shanghai Jining Biological Technology Co., Ltd, Shanghai, China). The cells were maintained in RPMI-1640 medium (Gibco, Thermo Fisher Scientific-CN, Shanghai, China) supplemented with $10 \%$ fetal bovine serum (WISENT, Nanjing, China) and $1 \%$ penicillin-streptomycin (HyClone, Logan, UT, USA) in a humidified atmosphere $\left(5 \% \mathrm{CO}_{2}\right.$ at $\left.37^{\circ} \mathrm{C}\right)$. The medium was replaced every 2 days until the cells reached $80 \%$ confluence. PA (Sigma-Aldrich, St. Louis, MO, USA) was conjugated with fatty acid-free bull serum albumin (BSA) at a ratio of $3.3: 1$ at $37^{\circ} \mathrm{C}$. Stock solutions of $500 \mathrm{mM}$ PA were filtered with a $0.22-\mu \mathrm{m}$ pore size membrane and then stored at $-20^{\circ} \mathrm{C}$ for use.

\section{Western blotting analysis}

RAECs were washed with ice-cold phosphate-buffered saline (PBS) and scraped in cell lysis buffer (Beyotime, Shanghai, China) for 15 $\min$ at $4^{\circ} \mathrm{C}$. The samples were centrifuged $\left(9600 \times g, 10 \mathrm{~min}, 4^{\circ} \mathrm{C}\right)$, and the proteins in the supernatant were quantified by a BCA Protein Quantification Kit (BU Technology Co., Ltd, Nanjing, China). After boiling for $5 \mathrm{~min}$ at $95^{\circ} \mathrm{C}$ in $1 \times$ loading buffer (BU Technology Co., Ltd.), $30 \mu \mathrm{g}$ total protein was separated by $10 \%$ sodium dodecyl sulfate-polyacrylamide gel electrophoresis (SDSPAGE) at $80 \mathrm{~V}$ for $30 \mathrm{~min}$ and $120 \mathrm{~V}$ for $1 \mathrm{~h}$ and then transferred to polyvinylidene fluoride membranes $(0.45 \mu \mathrm{m}$, Millipore Co., Ltd., Boston, MA, USA) for $1 \mathrm{~h}$ at $250 \mathrm{~mA}$. The membranes were blocked with nonfat milk (5\% w/v) in Tris-buffered saline with Tween-20 for 90 min. After washing three times with TBST, the membranes were probed with primary antibodies against SOCS3 (catalog no. 140251-AP, 1:500, Proteintech, Wuhan, China), Ob-Rb (catalog no. 209661-AP, 1:1000, Proteintech), ASM (catalog no. 14609-1-AP, 1:500, Proteintech), extracellular signal-regulated kinase 1/2 (ERK1/2) (catalog no. AF6240, 1:2000, Affinity, Cincinnati, OH, USA), p-ERK1/ 2 (Thr202/Tyr204) (catalog no. AF1014, 1:2000, Affinity), STAT3 (catalog no. AF6294, 1:2000, Affinity), phosphorylated (p)-STAT3 (Tyr705) (catalog no. AF3293, 1:1000, Affinity), Akt (catalog no. 10176-2-AP, 1:1000, Proteintech), p-Akt (Ser473) (catalog no. CY6016, 1:1000, Abways, Shanghai, China), eNOS (catalog no. AF0096, 1:1000, Affinity), and p-eNOS (Ser1177) (catalog no. AF3247, 1:1000, Affinity) overnight at $4{ }^{\circ} \mathrm{C}$. $\beta$-Actin (catalog no. AF7018, 1:5000, Affinity) and $\beta$-tubulin (catalog no. AB0039, 1:5000, Abways) were used as control proteins. After washing three times, the membranes were incubated with goat anti-rabbit immunoglobulin $\mathrm{G}(\mathrm{IgG})(\mathrm{H}+\mathrm{L})$ horseradish peroxidase (HRP) (catalog no. AB0101, 1:5000, Abways) for $2 \mathrm{~h}$. The blots were detected by a chemiluminescent detection system with High-sig ECL Western Blotting Substrate (1:1, Tanon, Shanghai, China). Densitometric analysis of the images was performed with ImageJ software (NIH, Littleton, $\mathrm{CO}$, USA).

RNA interference of ASM and Ob-Rb siRNAs targeting ASM and Ob-Rb were purchased from Santa Cruz (sc-270548, Santa Cruz, CA, USA) and Sango Biotech (RS5491, Shanghai, China), respectively. The targeting sequences of the
ASM and Ob-Rb siRNAs were 5'-GCAGGUCACACUUGCUGUTT-3' and 5'-GGAUGAAUCUAGCCGAGAATT-3', respectively. A scrambled small RNA (5'-AAUUCUCCGAACGUGUCACGU-3') was confirmed to be a nonsilencing double-stranded RNA and was used as a control in the current study. Transfection of the siRNAs and scrambled RNA was performed using the Lipofectamine ${ }^{T M} 2000$ Transfection Reagent (Thermo Fisher Scientific-CN) according to the manufacturer's instructions. The effectiveness of the siRNAs targeting ASM and $\mathrm{Ob}-\mathrm{Rb}$ were confirmed by Western blot analysis.

\section{ASM activity assay}

ASM activity in RAECs and serum was measured with an Amplite ${ }^{T M}$ Fluorimetric Acidic Sphingomyelinase Assay Kit (Thermo Fisher Scientific-CN) according to the manufacturer's instructions. In brief, the cellular extracts of RAECs were diluted in $50 \mathrm{mM}$ sodium acetate buffer $(\mathrm{pH}=5)$. After adequate incubation, the samples were mixed with corresponding working solutions. The fluorescence intensity was monitored with a fluorescence microplate reader at $\mathrm{Ex} / \mathrm{Em}=540 \mathrm{~nm} / 590 \mathrm{~nm}$.

\section{Immunofluorescence assay for ceramide}

Immunofluorescence of ceramide was measured according to the methods described by Li et al. [11] and Palau et al. [13]. RAECs were plated in a six-well plate. After treatment, the cells were washed twice with PBS and fixed with 4\% paraformaldehyde for $15 \mathrm{~min}$ and then blocked with 5\% BSA in PBS for $1.5 \mathrm{~h}$. The cells were incubated with an anti-ceramide antibody (1:20, Enzo, New York City, NY, USA) overnight at $4{ }^{\circ} \mathrm{C}$ and then incubated with goat anti-mouse $\operatorname{lgG}(\mathrm{H}+\mathrm{L})$ fluorescein isothiocyanate (FITC) (1:100, Abways) for $2 \mathrm{~h}$. Before analysis by fluorescence microscopy, the cells were washed three times with PBS and incubated with 4',6-diamidino-2-phenylindole in PBS for $15 \mathrm{~min}$. The mean fluorescence intensity was used to show the level of ceramide in RAECs.

Measurement of eNOS activity and NO levels

eNOS activity and NO levels in cellular extracts and serum were measured with commercially available kits (Nanjing Jiancheng Bioengineering Institute, Nanjing, China) according to the manufacturer's instructions. NOS catalyzes the reaction between $L$-arginine ( $L$-Arg) and molecular oxygen to form NO, and then NO reacts with nucleophilic substances to form colored compounds. A total of $100 \mu \mathrm{L}$ supernatant was incubated with $200 \mu \mathrm{L}$ substrate buffer, $10 \mu \mathrm{L}$ reaction accelerator, and $100 \mu \mathrm{L}$ color development reagent in a $37^{\circ} \mathrm{C}$ water bath for $15 \mathrm{~min}$ after mixing sufficiently. Then, $100 \mu \mathrm{L}$ clearing reagent and $2 \mathrm{~mL}$ termination buffer were added and mixed sufficiently. The absorbance was read at $530 \mathrm{~nm}$ using a microplate reader (POLARstar Omega ${ }^{\circledR}$, BMG LABTECH, Offenburg, Germany). One NOS enzymatic unit was defined as the capacity of forming $1 \mathrm{nmol} \mathrm{NO}$ in $1 \mathrm{~min}$ per milligram of protein. eNOS activity was determined by subtracting inducible NOS activity from total NOS activity, and the results were normalized to the mean value of the control. NO is oxidized by oxygen to nitrate and nitrite, which react with a chromogenic agent to generate azo dyes and can be measured spectrophotometrically. A total of $160 \mu \mathrm{L}$ supernatant was added to the reaction buffer and incubated at $37^{\circ} \mathrm{C}$ for $15 \mathrm{~min}$, and the reaction was stopped by adding termination buffer. The absorbance was read at $550 \mathrm{~nm}$ using a microplate reader (POLARstar Omega ${ }^{\circledR}$, BMG Labtech, Offenburg, Germany).

\section{Co-immunoprecipitation}

RAECs were washed three times with PBS and scraped in cell lysis buffer (Beyotime) for $15 \mathrm{~min}$ at $4{ }^{\circ} \mathrm{C}$. The samples were centrifuged $\left(14000 \times 9,15 \mathrm{~min}, 4^{\circ} \mathrm{C}\right)$, and the supernatant was mixed with lgG antibody and protein $\mathrm{A}+\mathrm{G}$ agarose. After incubation for $1 \mathrm{~h}$ at $4^{\circ} \mathrm{C}$ and centrifugation at $600 \times g$ for $5 \mathrm{~min}$, the total proteins were incubated with $\mathrm{p}$-STAT3 or p-Akt antibodies overnight at $4{ }^{\circ} \mathrm{C}$. The 
protein $\mathrm{A}+\mathrm{G}$ agarose was added to the extracts for $2 \mathrm{~h}$ at $4{ }^{\circ} \mathrm{C}$. The precipitated materials were obtained by centrifugation $(860 \times g$, $30 \mathrm{~s}$ ), washed five times with $1 \mathrm{~mL}$ lysis buffer, and then resuspended in $1 \times$ SDS loading buffer for Western blotting analysis.

Establishment of rat model with leptin-resistant models

Adult male Sprague-Dawley (SD) rats weighing 200-250 g were purchased from Qinglongshan Lab Animal Ltd. (Nanjing, China). Animal handling and experimental procedures were approved by the ethics committee of China Pharmaceutical University and conducted according to the NIH Guide for the Care and Use of Laboratory Animals.

Male SD rats were used to generate a leptin-resistant model according to the methods described by Yuan et al. [14]. SD rats were fed a HFD ( $22 \mathrm{~g}$ per day) containing $5 \times 10 \mathrm{kcal} / \mathrm{g}$ with $60 \%, 19 \%$, and $21 \%$ calories from fat, protein, and carbohydrates, respectively. Both the normal chow and high-fat diet were purchased from Qinglongshan Lab Animal Ltd. The rats were divided into four groups: the control group (regular chow, $n=6)$, untreated HFD-fed group $(n=6)$, the HFD-fed group treated with AMI $\left(10 \mathrm{mg} \cdot \mathrm{kg}^{-1}\right.$ per day, intraperitoneally (i.p.), $n=6)$, and the HFD-fed group treated with IMI $\left(10 \mathrm{mg} \cdot \mathrm{kg}^{-1}\right.$ per day, i.p., $n=6$ ).

For the first 9 weeks, the rats in the untreated group were fed a HFD, and the rats in the control group were given regular chow. During weeks 10-11, interventions with $\mathrm{AMI}$ and IMI were conducted for 2 weeks $[15,16]$, and an equal volume of saline solution was given to the rats in the control group and untreated group. Body weight was monitored once a week.

Serum biochemical measurements

Blood samples from the rats were collected and centrifuged at $600 \times g$ for $10 \mathrm{~min}$. The serum was collected and stored at $-80^{\circ} \mathrm{C}$ before use. The leptin concentration was determined by a rat leptin ELISA Kit (SenBeiJia Biotechnology Co., Ltd, Nanjing, China). The total cholesterol (T-CHO), triglyceride (TG), low-density lipoprotein (LDL), high-density lipoprotein (HDL), and nonesterified fatty acid (NEFA) levels were assayed following the instructions of the kits provided by the Nanjing Jiancheng Bioengineering Institute (Nanjing, China).

\section{Recording of isometric vascular tone}

Rats were anesthetized with $10 \%$ chloral hydrate, and then the thoracic aorta was quickly removed and immersed in Krebs-Henseleit solution $(\mathrm{KH}$, in $\mathrm{mM}, \mathrm{pH} 7.4,119.0 \mathrm{NaCl}, 25.0$ $\mathrm{NaHCO}_{3}, 11.1$ glucose, $2.4 \mathrm{CaCl}_{2}, 4.7 \mathrm{KCl}, 1.2 \mathrm{KH}_{2} \mathrm{PO}_{4}, 1.2 \mathrm{MgSO}_{4}$, and $0.024 \mathrm{Na}_{2}$ EDTA). The aortas were freed from connective tissue and fat and then cut into separated thoracic aortic rings with a width of $\sim 2 \mathrm{~mm}$. All anatomical procedures were carefully performed to protect the endothelium from accidental damage. The aortic rings were then suspended in water-jacketed tissue baths and tested for isometric force recorded by a PowerLab system (Chengdu Taimeng Co., Ltd, Chengdu, China). The KH solution was maintained at $37^{\circ} \mathrm{C}$, and mixed gas containing $95 \%$ $\mathrm{O}_{2}$ and $5 \% \mathrm{CO}_{2}$ was continuously bubbled through the bath. The baseline load placed on the aortic rings was $2.0 \mathrm{~g}$. Changes in isometric tension were recorded using a force displacement transducer (Chengdu Taimeng Co., Ltd). The aortic rings were washed every $10 \mathrm{~min}$, and after three equilibrations, the aortic rings were contracted with phenylephrine (Phe, $1 \mu \mathrm{M})$ to obtain a maximal response. Then, a cumulative dose-response curve of the thoracic aorta to acetylcholine (ACh, $\left.1 \times 10^{-9}-1 \times 10^{-4} \mathrm{M}\right)$, sodium nitroprusside (SNP, $\left.1 \times 10^{-9}-1 \times 10^{-4} \mathrm{M}\right)$, and leptin $\left(1 \times 10^{-9}-1 \times 10^{-4} \mathrm{M}\right)$ was obtained. The area under the curve (AUC) of the contractile response was calculated to represent the reactivity of the vascular endothelium to ACh, SNP, and leptin. In addition, the aortic rings were preincubated with $L-\operatorname{Arg}\left(10^{-4} \mathrm{M}\right.$,
Biosharp) or L-NG-nitroarginine (L-NNA, $10^{-4} \mathrm{M}$, Solarbio Biotechnology Co., Ltd, Beijing, China), and the contractile response to Phe $\left(1 \times 10^{-10}-1 \times 10^{-4} \mathrm{M}\right)$ among four groups were recorded. The change in the AUC ( $\triangle A U C)$ of the contractile response was calculated to represent the inducible or basal release of endothelial NO.

\section{Immunohistochemistry}

Rat thoracic aortas were perfused with PBS and fixed with $4 \%$ paraformaldehyde and embedded in paraffin. The paraffin sections of the aortic rings were sliced, dewaxed, and washed with PBS three times for 5 min, blocked with $3 \% \mathrm{H}_{2} \mathrm{O}_{2}-\mathrm{CH}_{3} \mathrm{OH}$ for $30 \mathrm{~min}$, and incubated in citrate antigen retrieval solution for 18 min and 5\% BSA in PBS for $1 \mathrm{~h}$ at room temperature. For immunohistochemical studies, the sections were incubated with an anti-ASM (1:200, Proteintech), anti-Ob-Rb (1:200, Proteintech), anti-SOCS3 (1:300, Proteintech) or anti-p-STAT3 (Tyr705) (1:200, Affinity) antibody overnight at $4{ }^{\circ} \mathrm{C}$. After washing three times with $\mathrm{PBS}$, the sections were incubated with goat anti-rabbit $\lg G(\mathrm{H}+\mathrm{L})$ $\operatorname{HRP}$ (1:100, Abways) for $2 \mathrm{~h}$ at room temperature. After washing three more times, the sections were incubated with diaminobenzidine (Beyotime) for 5 min for staining, washed with distilled water, and stained with hematoxylin for $5 \mathrm{~min}$.

For immunofluorescence staining for SOCS3 and ASM in the endothelium, the sections were incubated with an anti-ASM (1:200, Proteintech) or anti-SOCS3 (1:300, Proteintech) antibody overnight at $4{ }^{\circ} \mathrm{C}$ and then incubated with a goat anti-rabbit IgG $(\mathrm{H}+\mathrm{L})$ secondary antibody or Cy3 conjugate (1:100, Boster, Wuhan, China) for $2 \mathrm{~h}$ at room temperature. Then, the sections were incubated with anti-von Willebrand factor (vWF) (1:100, Abways) overnight at $4{ }^{\circ} \mathrm{C}$, followed by goat anti-rabbit lgG $(\mathrm{H}+\mathrm{L})$ Alexa Fluor 488 (1:100, Abways) for $2 \mathrm{~h}$ at room temperature. Finally, the sections were sealed with neutral balsam (Solarbio Biotechnology Co., Ltd.) and examined under an OLYMPUS IX53 microscope.

\section{Statistical analysis}

Statistical analysis was performed with IBM SPSS Statistics 20 software. The data are presented as the means \pm SEM. Significant differences between and within multiple groups were examined using ANOVA for repeated measures followed by Duncan's multiple-range test. An independent samples $t$ test was used to detect significant differences between two groups. $P<0.05$ was considered statistically significant.

\section{RESULTS}

ASM downregulation improved PA-induced leptin resistance in RAECs

PA, the most abundant saturated fatty acid found in animals and the human body, has been used to induce leptin resistance in vitro [17]. However, it is unclear whether PA can induce resistance in RAECs. SOCS3, p-STAT3, and Ob-Rb are regarded as the main indicators of leptin resistance $[18,19]$. Leptin can bind to $\mathrm{Ob}-\mathrm{Rb}$ to enhance the expression of $\mathrm{Ob}-\mathrm{Rb}$ and downstream signaling proteins, such as SOCS3. RAECs were incubated with PA for $24 \mathrm{~h}$, and $100 \mathrm{nM}$ leptin was added for $15 \mathrm{~min}$ prior to collecting cells. We found that PA dose-dependently increased the expression of SOCS3 and decreased the expression of Ob-Rb in RAECs treated with or without leptin (Fig. 1a, b). At a dose of $0.3 \mathrm{mM}, \mathrm{PA}$ had the strongest effects on the expression of SOCS3 and Ob-Rb. Thus, $0.3 \mathrm{mM}$ PA was used to induce leptin resistance in RAECs.

To investigate whether ASM is regulated by PA in RAECs, we measured the protein expression of ASM by Western blotting and ASM activity with an Amplite ${ }^{T M}$ Fluorimetric Acidic Sphingomyelinase Assay Kit. Relative to those in the control group, the protein expression and activity of ASM were significantly increased by PA 
a

$\mathrm{Ob}-\mathrm{Rb}-\longrightarrow-\cdots--100 \mathrm{kDa}$

$\beta$-Tubulin- $----0-50 \mathrm{kDa}$

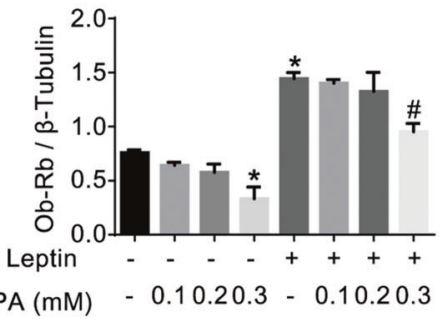

d

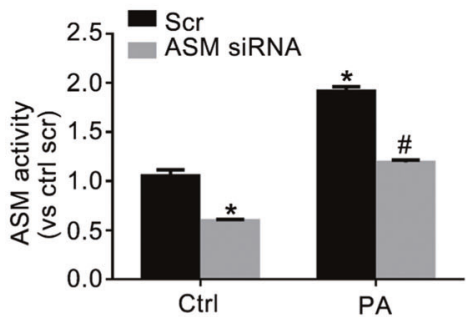

g

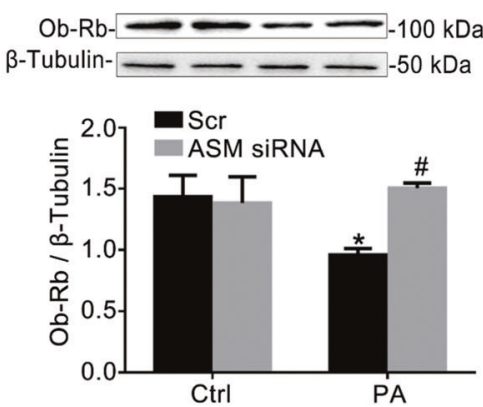

b

SOCS3- - - $-25 \mathrm{kDa}$ ß-Tubulin-

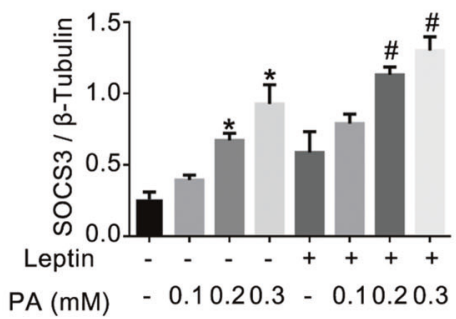

e

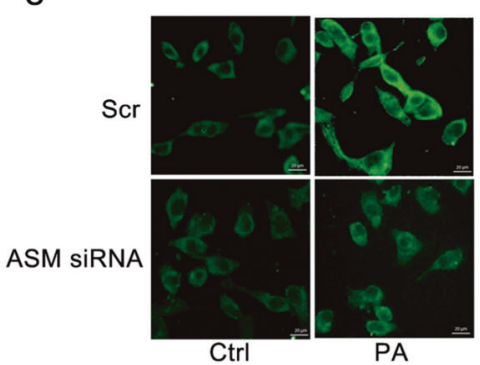

h
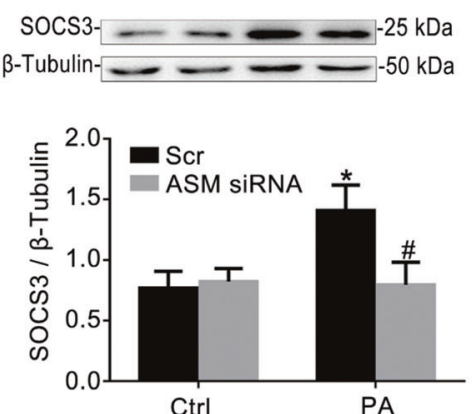

C
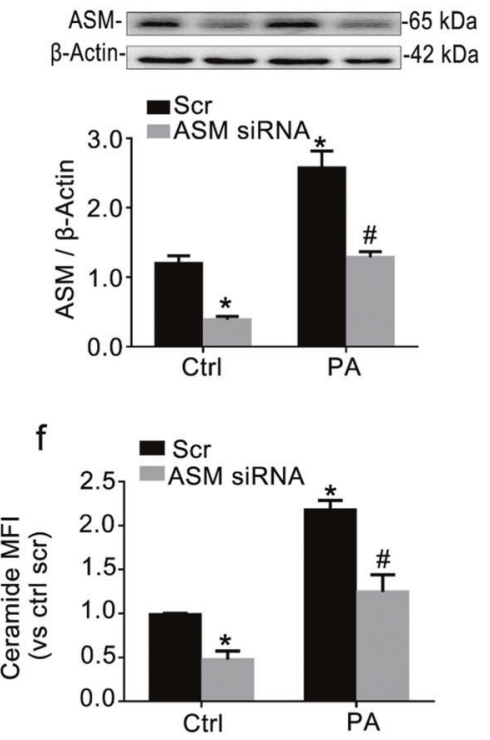

i
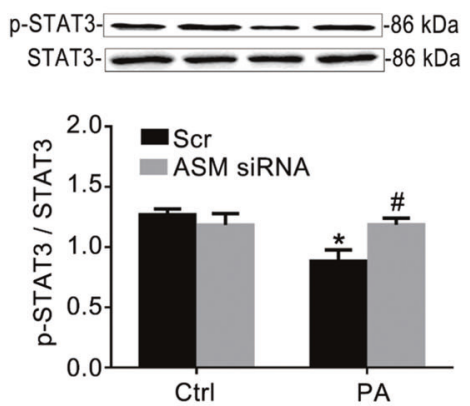

Fig. 1 ASM downregulation improved PA-induced leptin resistance in RAECs. Western blot gels and summarized data showing the protein expression of Ob-Rb (a) and SOCS3 (b) in RAECs treated with PA $(0.1,0.2$, and $0.3 \mathrm{mM})$ with or without $100 \mathrm{nM}$ leptin. RAECs were incubated with $0.3 \mathrm{mM}$ PA for $24 \mathrm{~h}$ and were then transfected with ASM siRNA for $24 \mathrm{~h}$. Then, $100 \mathrm{nM}$ leptin was added for $15 \mathrm{~min}$ prior to the collection of the cells. Then, Western blotting and an Amplite ${ }^{T M}$ Fluorimetric Kit were used to test the protein expression of ASM (c) and ASM activity (d), respectively, in RAECs. Representative fluorescence images (e, scale bar $=20 \mu \mathrm{m}$ ) and summarized data showing the mean fluorescent intensity (MFI) of a FITC-labeled anti-ceramide antibody (f). The photographs were taken at $\times 400$ magnification. The protein expression of Ob$\mathrm{Rb}(\mathbf{g})$ and SOCS3 $(\mathbf{h})$ and the ratio of p-STAT3/STAT3 (i) was determined using Western blotting. The data are the means \pm SEMs from three experiments. ${ }^{*} P<0.05$ vs. scramble control (Scr Ctrl); ${ }^{\#} P<0.05$ vs. the PA alone-treated group.

(Fig. 1c, d). Meanwhile, the transfection of ASM siRNA into RAECs resulted in remarkable inhibition of the protein expression and activity of ASM, which confirmed the effectiveness of ASM siRNA transfection in the current study. ASM catalyzes the hydrolysis of sphingomyelin to produce ceramide. Here, according to the methods described by Li et al. [11], Palau et al. [13] and Maroni et al. [20], RAECs were incubated with an anti-ceramide antibody followed by goat anti-mouse IgG-FITC. As shown in Fig. 1 e, $f(\times 400$ magnification), the fluorescence in PA-treated RAECs was more obvious than that in the control group, suggesting that ceramide production was increased by PA. It has been demonstrated that the surface accumulation of ceramides on endothelial cells usually reflects ASM activity [21]. As expected, ASM siRNA transfection also decreased PA-induced ceramide production in RAECs. Importantly, the downregulation of ASM by ASM siRNA increased the expression of $\mathrm{Ob}-\mathrm{Rb}$ and the phosphorylation of STAT3 at Tyr705 and decreased the expression of SOCS3 (Fig. 1g-i). Moreover, ASM overexpression increased the expression of SOCS3 and decreased the expression of $\mathrm{Ob}-\mathrm{Rb}$ and the phosphorylation of STAT3 (Supplemental Fig. S1). Thus, ASM indeed regulated leptin resistance in RAECs.
ASM inhibition attenuated leptin resistance in Ob-Rb-silenced RAECs

A reduction in Ob-Rb signal transduction effectiveness is an important cause of leptin resistance [18]. It has also been reported that $\mathrm{Ob}-\mathrm{Rb}$ gene silencing decreases the expression of p-STAT3 and increases the expression of SOCS3 [22]. Thus, in the current study, we used Ob-Rb gene silencing to block leptin signal transduction to imitate leptin resistance in RAECs. Ob-Rb siRNA was demonstrated to have a maximum interference efficiency of $\sim 63 \%$ (Fig. 2a). SOCS3 expression was increased, and STAT3 phosphorylation at Tyr705 was decreased in RAECs transfected with Ob-Rb siRNA relative to controls (Fig. 2b, c). Thus, RAECs with $\mathrm{Ob}-\mathrm{Rb}$ gene knockdown showed leptin resistance. AMI $(10 \mu \mathrm{M})$ and IMI $(10 \mu \mathrm{M})$ were chosen as inhibitors of ASM activity for the in vitro study. We found that AMI and IMI, compared with Ob-Rb siRNA lone, elevated STAT3 phosphorylation and decreased SOCS3 expression. Combined with the data presented in Fig. 1, these data suggested that ASM blockade improved leptin resistance in RAECs not only by regulating the expression of Ob$\mathrm{Rb}$ but also by directly affecting the phosphorylation of STAT3 and the expression of SOCS3. 
a
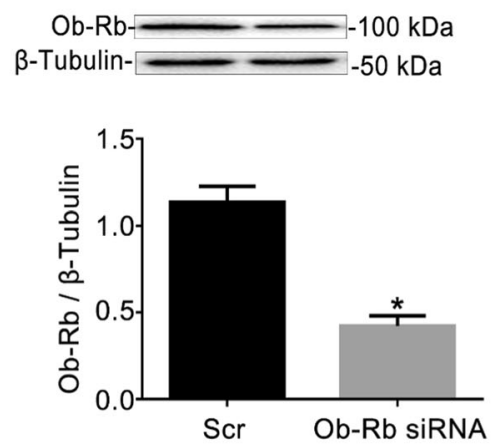

b

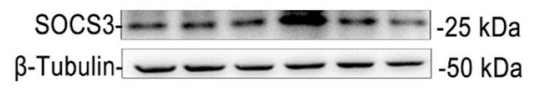

C

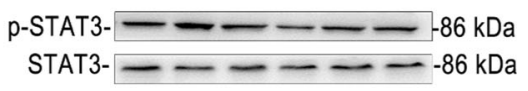

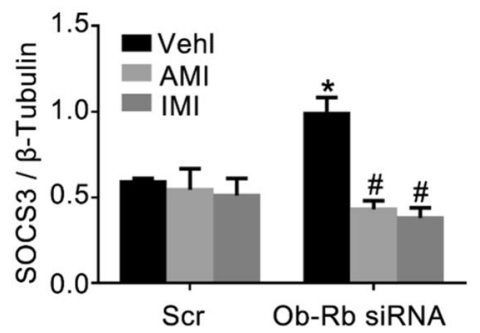

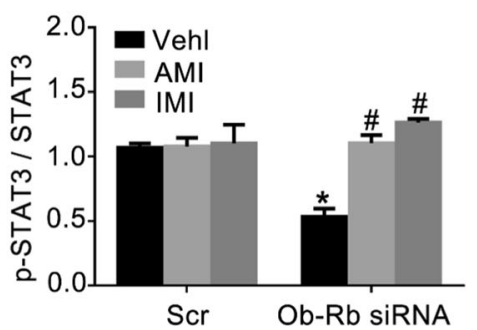

Fig. 2 ASM inhibition attenuated leptin resistance in Ob-Rb-silenced RAECs. Representative Western blot gels for Ob-Rb and summarized data showing the efficiency of Ob-Rb siRNA transfection (a). RAECs were transfected with Ob-Rb siRNA for $48 \mathrm{~h}$ and were treated with AMI (10 $\mu \mathrm{M})$ or IMI $(10 \mu \mathrm{M})$ for $24 \mathrm{~h}$. Then, $100 \mathrm{nM}$ leptin was added for 15 min prior to the collection of the cells. The protein expression of SOCS3 (b) and the ratio of $\mathrm{p}$-STAT3/STAT3 (c) were tested using Western blotting. The data are the means \pm SEMs from three experiments. ${ }^{*} P<0.05$ vs. vehicle control (Vehl Scr); ${ }^{\#} P<0.05$ vs. the Ob-Rb siRNA alone-transfected group.

a

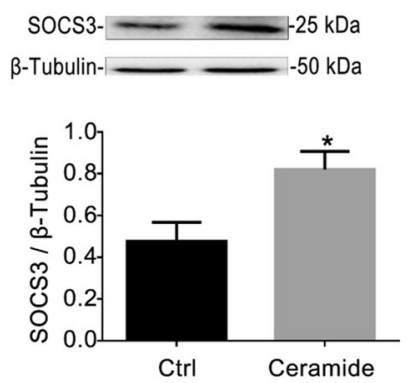

e
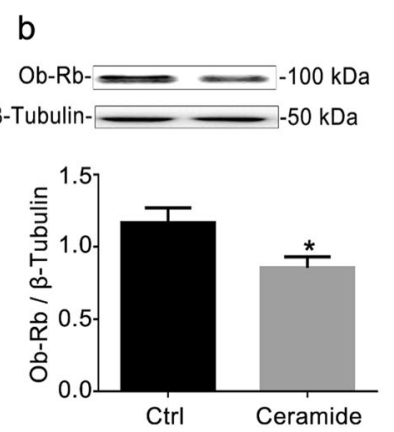

C
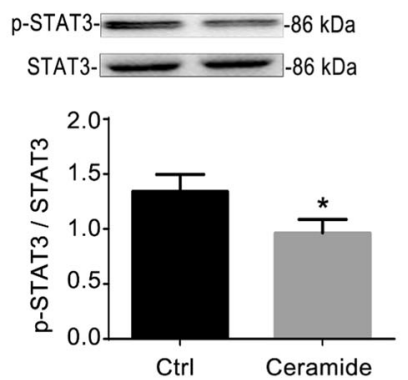

d
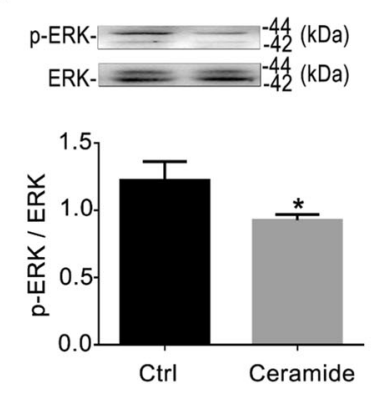

$f$

g

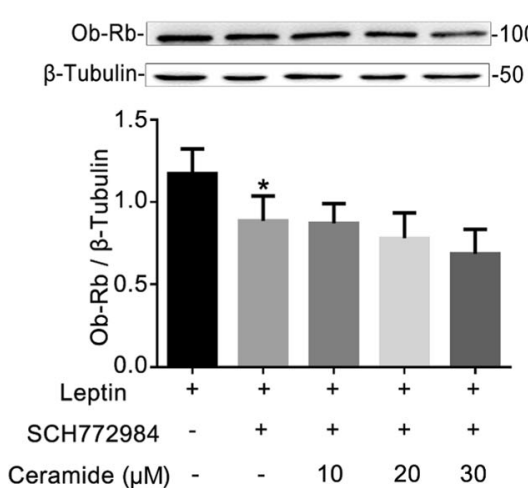

p-STAT3- $-\longrightarrow--16 \mathrm{kDa}$
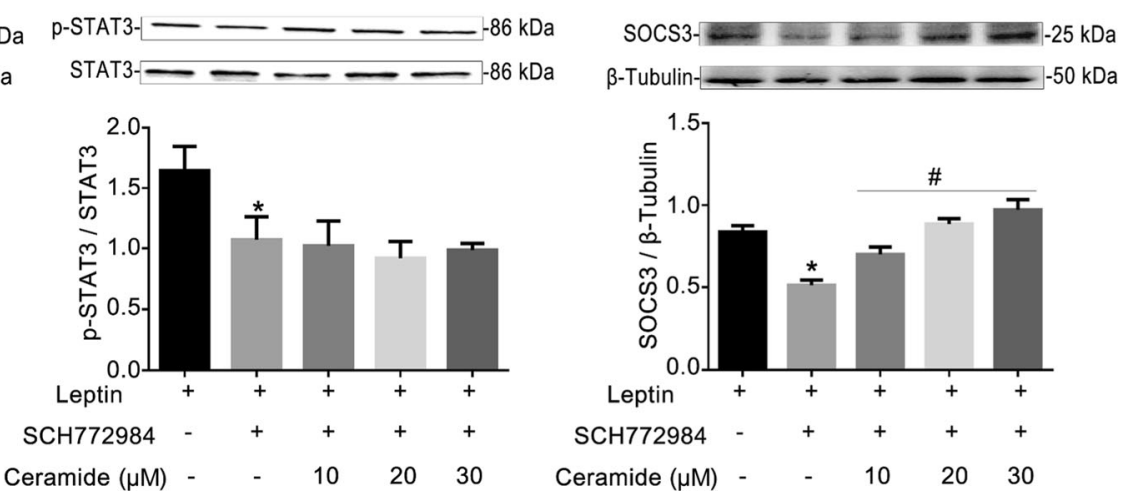

Fig. 3 Ceramide supplementation induced leptin resistance through ERK1/2. RAECs were incubated with $10 \mu \mathrm{M}$ ceramide, and $100 \mathrm{nM}$ leptin was added for $15 \mathrm{~min}$ prior to the collection of the cells. Representative Western blot gels and summarized data showing the protein expression of SOCS3 (a) and Ob-Rb (b) and the ratios of p-STAT3-Tyr705/STAT3 (c) and p-ERK/ERK (d) in RAECs. The protein expression of $\mathrm{Ob-Rb}(\mathbf{e})$ and SOCS3 (g) and the ratio of p-STAT3/ STAT3 (f) were evaluated in RAECs pretreated with $1 \mu \mathrm{M}$ SCH772984 for $24 \mathrm{~h}$ and then incubated with different doses of ceramide $(10,20,30 \mu \mathrm{M})$. The data are the means \pm SEMs from 3 experiments. ${ }^{*} P<0.05$ vs. control $(C t r l)$; ${ }^{\#} P<0.05$ vs. the SCH772984 alone-treated group.

Ceramide supplementation induced leptin resistance through ERK $1 / 2$

It has been reported that ceramide can induce an increase in the expression of SOCS3 [23]. To further confirm the regulation of leptin resistance by ASM, ceramide $(10 \mu \mathrm{M}, 15 \mathrm{~min}$, Enzo, USA) was administered to RAECs. As shown in Fig. $3 a-c$, the exogenous application of ceramide dramatically increased the protein of SOCS3 and decreased the protein expression of Ob-Rb and $\mathrm{p}$ STAT3 (Tyr705). ERK1/2 is involved in the regulation of the expression of leptin receptors [20]. As shown in Fig. 3d, it was found that the phosphorylation of ERK1/2 was significantly lower in RAECs responding to ceramide. RAECs were pretreated with the ERK1/2 activity inhibitor SCH772984 $(1 \mu \mathrm{M}, 24 \mathrm{~h}$, Abmole Bioscience Inc., USA) followed by different doses of ceramide $(10,20$, and $30 \mu \mathrm{M})$. Compared with SHC772984 alone, ceramide administration failed to further downregulate $\mathrm{Ob}-\mathrm{Rb}$ expression and STAT3 phosphorylation (Fig. 3e, f). In contrast, the expression of SOCS3 was upregulated by ceramide (Fig. $3 \mathrm{~g}$ ). These results indicated that ceramide inhibited Ob-Rb expression and STAT3 phosphorylation through ERK1/2 activation. However, ceramide increased the expression of SOCS3 independent of ERK1/2 activation. 
a

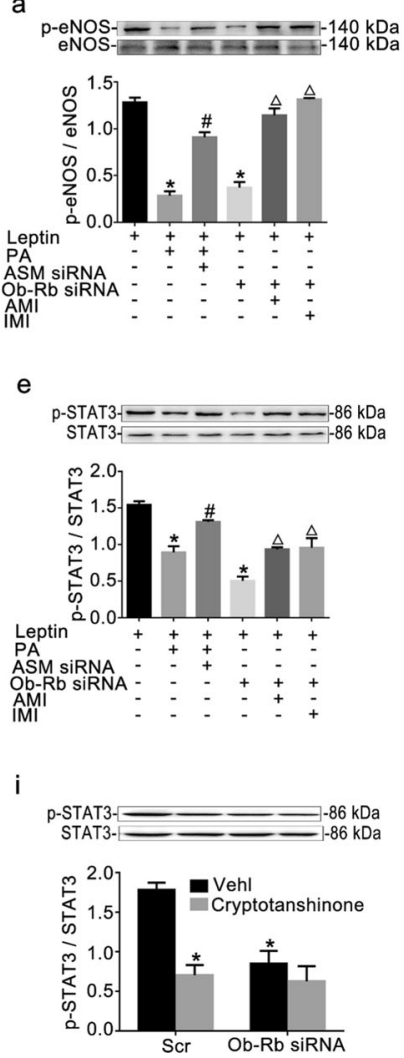

b

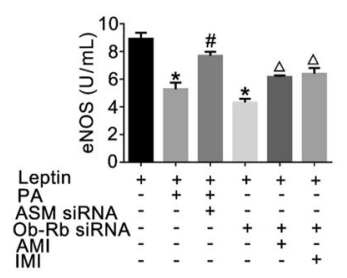

f
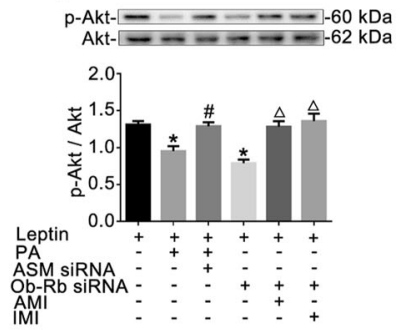

j $\begin{array}{r}\mathrm{p}-\mathrm{Akt}-\square- \\ \mathrm{Akt}-\square\end{array}-62 \mathrm{kDa}$

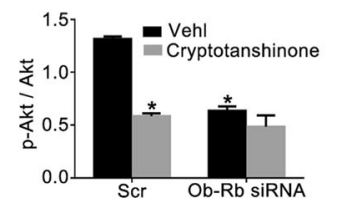

c

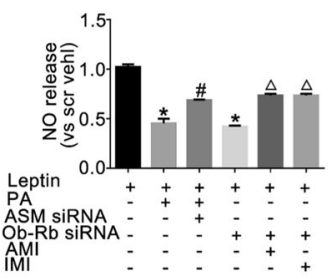

g
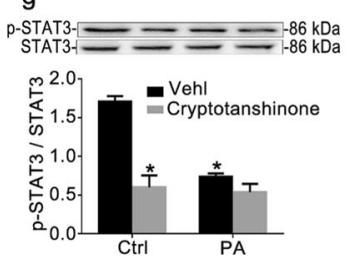

k

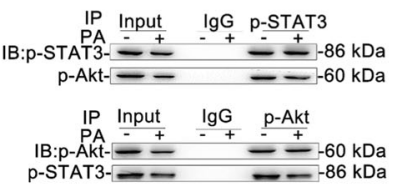

d

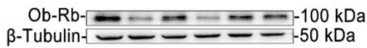

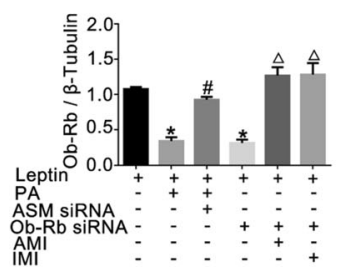

h

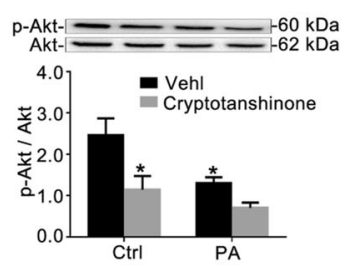

।

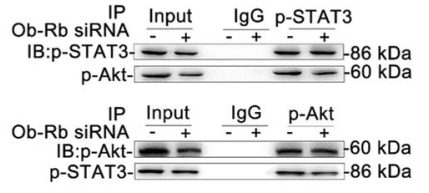

Fig. 4 ASM downregulation increased eNOS/NO signaling in RAECs. RAECs were incubated with $0.3 \mathrm{mM}$ PA for $24 \mathrm{~h}$ and then transfected with ASM siRNA, or RAECs were transfected with Ob-Rb siRNA for $48 \mathrm{~h}$ and then treated with $10 \mu \mathrm{M} \mathrm{AMI}$ and $10 \mu \mathrm{M}$ IMI for $24 \mathrm{~h}$. Then, $100 \mathrm{nM}$ leptin was added for $15 \mathrm{~min}$ prior to the collection of the cells. Summarized data showing the effects of ASM downregulation on the ratio of $p$ eNOS (Ser1177)/eNOS (a), eNOS activity (b), NO release (c), Ob-Rb expression (d), and the ratios of p-STAT3/STAT3 (e) and p-Akt/Akt (f) in RAECs. ${ }^{*} P<0.05$ vs. vehicle control (Vehl Scr); ${ }^{*} P<0.05$ vs. the PA alone-treated group; ${ }^{\Delta} P<0.05$ vs. the Ob-Rb siRNA alone-transfected group. Representative Western blot gels and summarized data showing the ratios of p-STAT3/STAT3 and p-Akt/Akt in RAECs that were pretreated with $10 \mu \mathrm{M}$ cryptotanshinone for $24 \mathrm{~h}$ and then incubated with $0.3 \mathrm{mM}$ PA for $24 \mathrm{~h}(\mathbf{g}, \mathbf{h})$ or transfected with Ob-Rb siRNA for $48 \mathrm{~h}(\mathbf{i}$, $\mathbf{j})$. ${ }^{*} P<0.05$ vs. vehicle control (Vehl Scr); ${ }^{\#} P<0.05$ vs. the $\mathrm{SCH} 772984$ alone-treated group. The co-immunoprecipitation assay was used to test the interaction between p-STAT3 and p-Akt in RAECs treated with PA (k) or transfected with Ob-Rb siRNA (I). The data are the means \pm SEMs from three experiments.

ASM downregulation increased eNOS/NO signal in RAECS Endothelial leptin resistance is related to endothelial dysfunction [17]. eNOS is a key enzyme that produces endothelial cell-derived $\mathrm{NO}$ and plays an important role in the regulation of endothelial function [24]. eNOS phosphorylation and activity (Fig. 4a, b) and the NO level (Fig. 4c) were decreased by PA incubation or Ob-Rb siRNA transfection and were effectively restored by ASM siRNA and ASM inhibitors, respectively. It has been reported that the serine/threonine protein kinase Akt can activate endothelial eNOS by directly phosphorylating serine 1177 , thereby leading to NO production [25]. As shown in Fig. 4f, the decreased phosphorylation of Akt under leptin resistance was reversed by ASM siRNA or ASM inhibitors. A similar trend was found for the expression of Ob$\mathrm{Rb}$ and the phosphorylation of STAT3 (Fig. 4d, e).

It remains unclear whether STAT3 regulates Akt during leptin signal transduction. Like PA incubation or Ob-Rb siRNA transfection, cryptotanshinone ( $10 \mu \mathrm{M}, 24 \mathrm{~h}$, Abmole Bioscience Inc., USA), an inhibitor of STAT3 phosphorylation at Tyr705, suppressed the phosphorylation of both STAT3 and Akt. However, cryptotanshinone had no additional inhibitory effect on the phosphorylation of STAT3 or Akt in RAECs subjected to PA incubation or Ob-Rb siRNA transfection (Fig. 4g-j). The co-immunoprecipitation assay showed that p-STAT3 and p-Akt were successfully immunoprecipitated under leptin resistance induced by PA incubation or Ob-Rb siRNA transfection, which further illustrated the interaction between STAT3 and Akt under leptin resistance (Fig. 4k, I).
AMI and IMI ameliorated abnormal metabolism in HFD-fed rats It has been demonstrated that AMI attenuates increases in plasma leptin concentrations, prevents weight gain, and improves dyslipidemia induced by a $\operatorname{HFD}[26,27]$. Following the consumption of a HFD, the body weight and weight gain of rats tended to increase (Fig. 5a, b). In addition, the levels of T-CHO, TG, LDL, and NEFA were significantly increased, and the level of $\mathrm{HDL}$ was significantly decreased in rats fed a HFD (Fig. 5c-g). These abnormal changes indicated the successful establishment of a model of obesity in rats and were dramatically reduced by intervention with $\mathrm{AMI}$ and IMI.

It has been suggested that HFD feeding can induce leptin resistance in rats [14]. Indeed, serum leptin concentration (Fig. 5h) and ASM activity (Fig. 5i) were significantly increased in HFD-fed rats compared with control rats. AMI and IMI inhibited the levels of serum leptin and ASM activity. In addition, reduced eNOS activity and $\mathrm{NO}$ production in the serum was observed in HFD-fed rats (Fig. 5j, k). Such abnormal NOS/NO signals were normalized by AMI and IMI treatment. Thus, ASM downregulation was related to the improvement of metabolic disorders, including systemic hyperleptinemia and NO production, in HFD-fed rats.

AMI and IMI relieved vascular leptin resistance in HFD-fed rats Immunohistochemical analysis revealed the expression of $\mathrm{Ob}-\mathrm{Rb}$, p-STAT3-Tyr705, SOCS3, and ASM in the arterial endothelium (Fig. 6a). In the control group, the definite visible precipitation of 


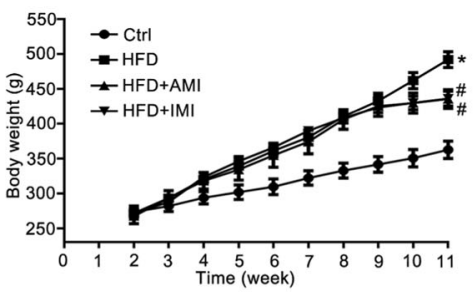

b

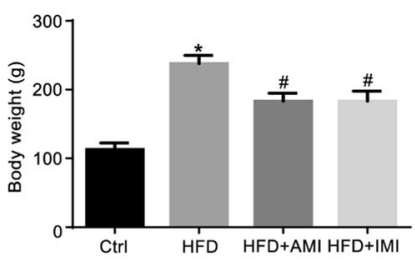

c

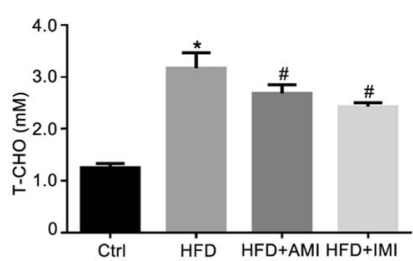

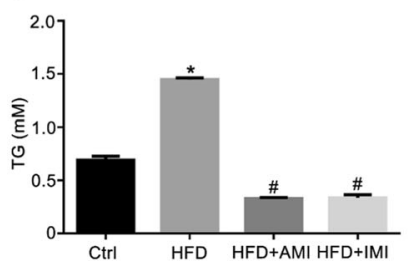

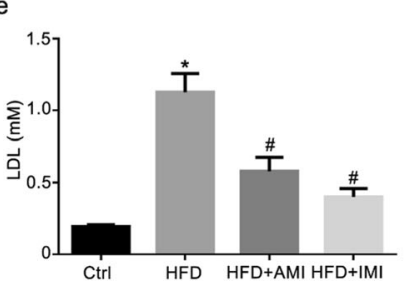

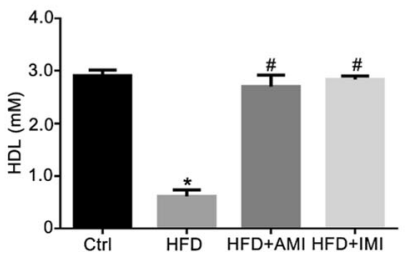

k

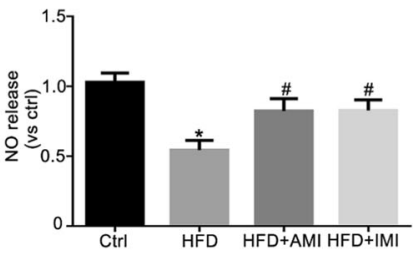

Fig. 5 AMI and IMI ameliorated abnormal metabolism in HFD-fed rats. Male Sprague-Dawley rats were fed a HFD to establish a rat model of leptin resistance. HFD-fed rats were treated with AMI $\left(10 \mathrm{mg} \cdot \mathrm{kg}^{-1}\right.$ per day, i.p.) and IMI (10 $\mathrm{mg} \cdot \mathrm{kg}^{-1}$ per day, i.p.) for 2 weeks. Summarized data showing the role of AMI and IMI on body weight (a), weight gain (b), T-CHO (c), TG (d), LDL (e), NEFA (f), HDL (g), and leptin levels (h), ASM activity (i), eNOS activity (j) and NO release (k) in the serum. The data are the means $\pm \mathrm{SEMs}$ from six experiments. ${ }^{*} P<0.05$ vs. control $\left(\right.$ Ctrl); ${ }^{\#} P<0.05$ vs. HFD-fed rats.

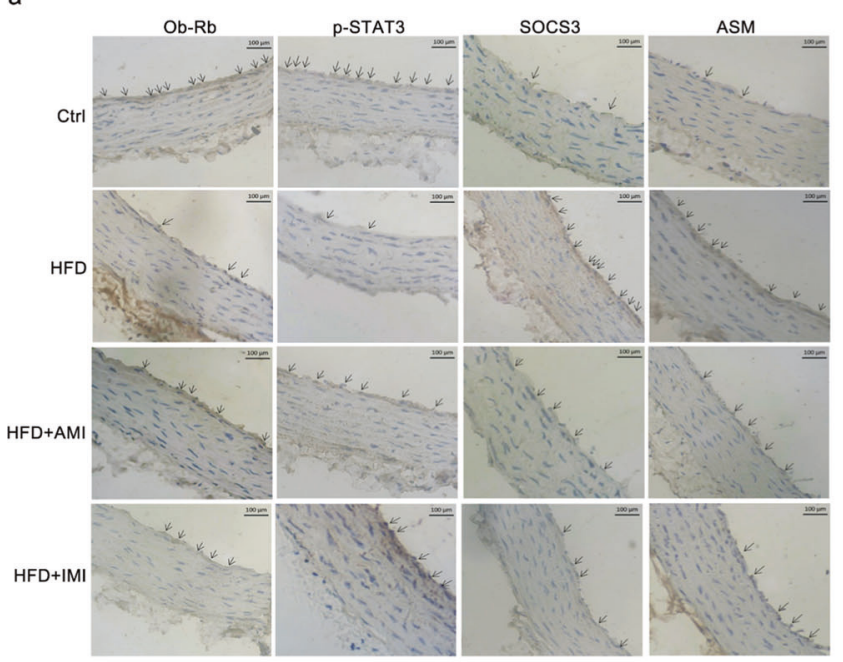

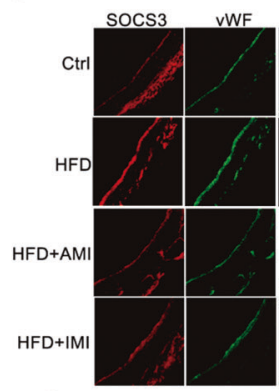
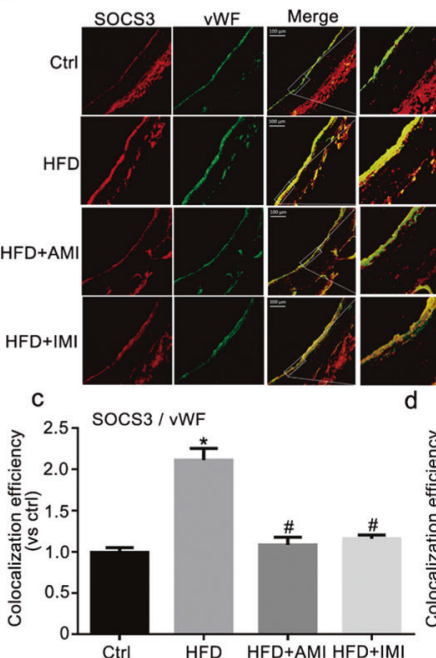
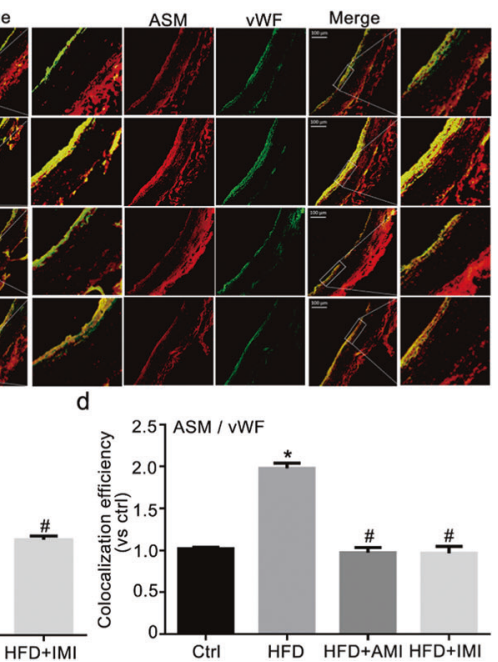

Fig. 6 AMI and IMI relieved endothelial leptin resistance in HFD-fed rats. Representative immunohistochemical images showing the role of AMI and IMI on the levels of Ob-Rb, p-STAT3-Tyr705, SOCS3, and ASM in the aorta (a, scale bar $=100 \mu \mathrm{m})$. Representative immunofluorescence images showing the colocalization of ASM or SOCS3 with vWF $(\mathbf{b}$, scale bar $=100 \mu \mathrm{m})$ in the vascular endothelium. The calculated colocalization efficiency is shown in Fig. $6 \mathrm{c}$, d. The data are the means \pm SEMs from six experiments. ${ }^{*} P<0.05$ vs. control (Ctrl); ${ }^{\#} P<0.05$ vs. HFD-fed rats.

Ob-Rb and p-STAT3 and the inconspicuous precipitation of SOCS3 and ASM were observed in the endothelium of the aorta. The weakened precipitation of Ob-Rb and p-STAT3 and the intensified precipitation of SOCS3 and ASM were noted in HFD-fed rats and were significantly reversed following intervention with $\mathrm{AMI}$ and IMI.
VWF is a well-recognized marker of endothelial cells. Based on confocal microscopy, the colocalization of SOCS3 or ASM with VWF, shown in yellow, in the aortas of HFD-fed rats, was apparently increased (Fig. 6b). The colocalization efficiency was calculated and is shown in Fig. $6 c$, d. This result suggested an increase in SOCS3 or ASM in the aortic endothelium due to HFD 
a

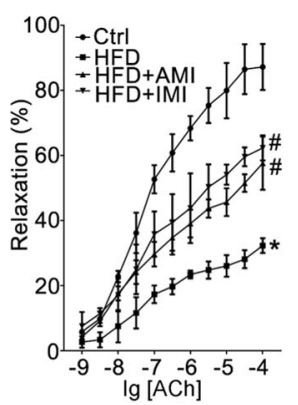

e

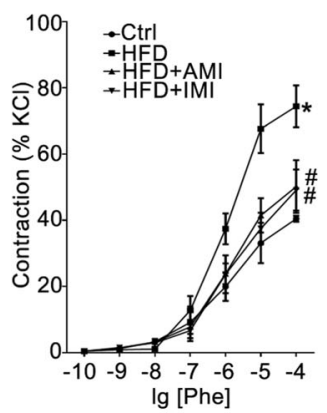

b

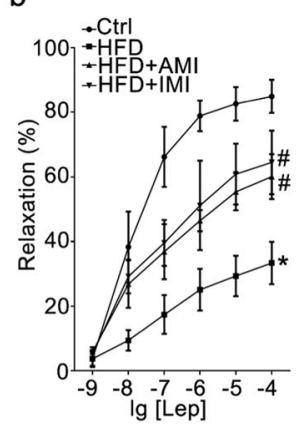

$f$

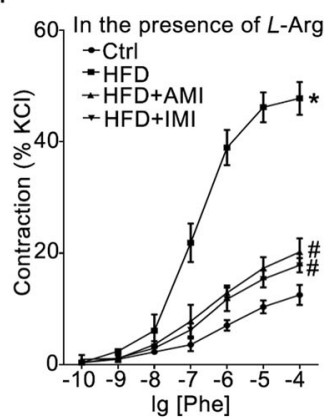

c

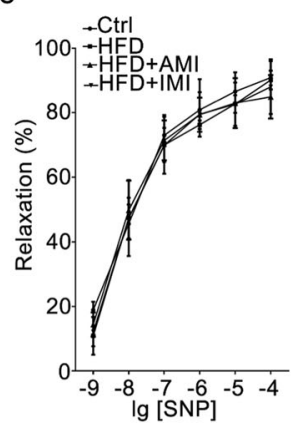

g

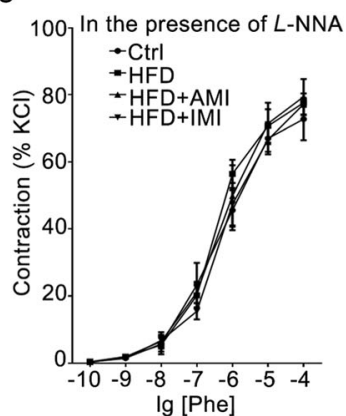

d

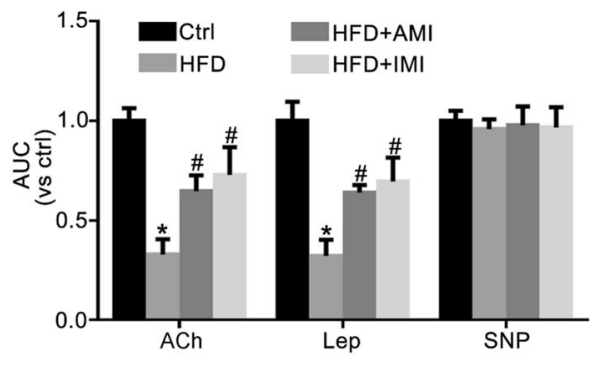

h

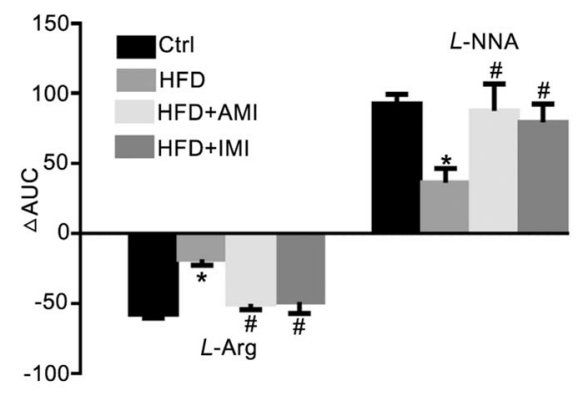

Fig. 7 AMI and IMI relieved vascular dysfunction in HFD-fed rats. Vascular responses to acetylcholine (ACh), leptin (Lep) and sodium nitroprusside (SNP) at a dose of $1 \times 10^{-9}-1 \times 10^{-4} \mathrm{M}$ were determined in the thoracic aorta (a-c). Summarized data showing the effect of AMI and IMI on the AUCs in response to ACh, Lep and SNP (d). Vascular concentration-response curves of the thoracic aorta to phenylephrine (Phe) in the absence (e) and presence of $L$-arginine $\left(L\right.$-Arg, $\left.1 \times 10^{-4} \mathrm{M}\right)(\mathbf{f})$ or $L$-NG-nitroarginine $\left(L\right.$-NNA, $\left.1 \times 10^{-4} \mathrm{M}\right)(\mathbf{g})$ were obtained. The $\triangle$ AUC of the contractile response to Phe represented the change in induced NO release and the basal NO level (h). The data are the means \pm SEMs from six experiments. ${ }^{*} P<0.05$ vs. control $\left(C\right.$ trl); ${ }^{\#} P<0.05$ vs. HFD-fed rats.

feeding. AMI and IMI almost completely blocked the expression of SOCS3 and ASM in the endothelium of HFD-fed rats. Combined with the in vivo results, these results demonstrated that the amelioration of endothelial leptin resistance was associated with the inhibition of ASM in the endothelium of the aorta by AMI and IMI.

\section{AMI and IMI improved vascular function in HFD-fed rats}

Leptin can induce dose-dependent vasorelaxation in the aorta [28]. Vascular responses of intact thoracic aortas from rats to $\mathrm{ACh}$ $\left(1 \times 10^{-9}-1 \times 10^{-4} \mathrm{M}\right)$, leptin (Lep, $\left.1 \times 10^{-9}-1 \times 10^{-4} \mathrm{M}\right)$, and SNP $\left(1 \times 10^{-9}-1 \times 10^{-4} \mathrm{M}\right)$ were tested. Compared to those in control rats, the endothelium-dependent relaxant responses to $\mathrm{ACh}$ and leptin were significantly impaired in HFD-fed rats and were obviously enhanced by treatment with $\mathrm{AMI}$ and IMI for 2 weeks (Fig. 7a, b). However, the vasorelaxation response to SNP showed no significant difference among the four groups, showing that vascular smooth muscles had the normal ability to relax (Fig. 7c). The AUC was calculated to show the effect of AMI and IMI on the diastolic function of the thoracic aorta as previously described [29], indicating that AMI or IMI treatment increased the AUC of the HFDfed group in response to ACh and leptin but not to SNP (Fig. 7d).

The concentration-response curves of aortic rings from the four groups to phenylephrine are shown in Fig. 7e. After preincubation with the NO synthesis substrate L-Arg, the dose response to phenylephrine was weakened in intact thoracic aortic rings (Fig. 7f). Conversely, after incubation with the NOS inhibitor L-NNA, the contractile response to phenylephrine was strengthened (Fig. $7 \mathrm{~g}$ ). The lower $\triangle \mathrm{AUC}$ in the HFD-fed group relative to the control is illustrated in Fig. 7h. The decrease in $\triangle A U C$ was significantly reversed by $\mathrm{AMI}$ and IMI treatment, suggesting that $\mathrm{AMI}$ and IMI treatment effectively improved NO release under basal and inducible conditions. Combined with the data in Figs. 5 and 6, these data suggested that AMI and IMI improved endothelial dysfunction by regulating blood lipid and leptin levels and inhibiting ASM activity in the aortic endothelium.

\section{DISCUSSION}

In the present study, we provided novel evidence that the downregulation of ASM/ceramide was critical for suppressing leptin resistance in RAECs triggered by PA incubation or Ob-Rb siRNA transfection. Simultaneously, we demonstrated that the inhibition of ASM with gene silencing or specific inhibitors improved eNOS/NO signaling and consequently alleviated the vascular dysfunction caused by endothelial leptin resistance.

Leptin, a $16-\mathrm{kDa}$ protein primarily secreted by adipocytes, regulates energy homeostasis via a so-called central mechanism in the brain. In the field of cardiovascular research, a physiological dose of leptin improves endothelial function, whereas large physiological doses cause endothelial dysfunction. This indicates that the regulation of leptin in endothelial function is bidirectional. A high concentration of leptin elevates SOCS3 expression and induces leptin resistance. Some pharmacologists have tried to develop leptin as a medicine for the treatment of diseases such as obesity and cardiovascular disease, but leptin resistance has restricted its clinical application [30,31]. The current study tried to detect new mechanisms of endothelial leptin resistance, which has become a hot topic in the treatment of atherosclerotic cardiovascular disease. The establishment of leptin resistance in endothelial cells allows us to further explore the potential physiological mechanisms and select effective drugs for endothelial leptin resistance. Elevated PA concentrations have been shown to induce leptin resistance and impair endothelial cell function associated with cardiovascular risk factors such as dyslipidemia and hypertension [12]. Although Ob-Rb initiates leptin signaling through more than seven different signal transduction pathways, excessive suppressor of SOCS3 activity is a potential mechanism of 
658

leptin resistance. SOCS3 binds to the Tyr985 residue of the leptin receptor and Janus kinase 2, inhibiting leptin-induced STAT3 [4]. In the current study, we demonstrated that the incubation of RAECs with PA, an abundant fatty acid, significantly promoted the protein expression of SOCS3 and inhibited the protein expression of Ob-Rb and p-STAT3 in RAECs, which was consistent with a study showing that PA can induce leptin resistance in human umbilical vein endothelial cells [17].

ASM is a key enzyme that catalyzes the breakdown of sphingomyelin to produce ceramide, which has been proposed to be linked to leptin resistance [32]. The current study found that the expression and activity of ASM was enhanced by PA in RAECs. This is consistent with a report showing PA-induced intracellular ceramide accumulation in N42 neuronal cells [33]. For the first time, we demonstrated that ASM overexpression increased the expression of SOCS3 and decreased the expression of $\mathrm{Ob}-\mathrm{Rb}$ and the phosphorylation of STAT3 and that the downregulation of ASM improved leptin sensitivity, which was associated with a decrease in SOCS3 expression and increases in Ob-Rb expression and STAT3 phosphorylation in RAECs.

Leptin receptors are broadly expressed in peripheral tissues. There are various published studies concerning the ability of leptin to control its own receptor isoforms, most of which have indicated that the downregulation of leptin receptors may explain the leptin resistance observed in obesity. For example, leptin downregulates the messenger RNA (mRNA) expression of both Ob-Rb and Ob-Ra within $24 \mathrm{~h}$ in HepG2 cells [34]. The mRNA levels of total Ob-R and specific Ob-Rb are decreased in testicular tissue taken from 30day-old rats and incubated for $120 \mathrm{~min}$ in the presence of recombinant leptin [35]. In contrast, the overexpression of the Ob$\mathrm{Rb}$ gene restores the phosphorylation of JAK2 and STAT3 in endothelial cells of Zucker fatty rats [36]. Transferring the Ob-Rb gene to leptin-resistant islets reverses diabetogenic phenotype [37]. Some studies have also found the downregulation of hypothalamic leptin receptor expression in the central nervous system under leptin resistance [38]. Importantly, mice deficient in leptin $(o b / o b)$ or its functional receptor $(d b / d b)$ develop morbid obesity, hyperphagia, and diabetes [39-41]. Based on these studies, $\mathrm{Ob}-\mathrm{Rb}$ downregulation is relevant to leptin resistance at the sites of peripheral action. Thus, we used $\mathrm{Ob}-\mathrm{Rb}$ gene silencing to establish a cell model mimicking leptin resistance in vitro. In the current study, the protein expression of SOCS3 was increased, and the protein expression of $\mathrm{Ob}-\mathrm{Rb}$ and $\mathrm{p}$-STAT3 was inhibited after transfection with $\mathrm{Ob}-\mathrm{Rb}$ siRNA. Thus, we believe that $\mathrm{Ob}-\mathrm{Rb}$ gene silencing contributes to leptin resistance in RAECs. ASM inhibitors attenuated leptin resistance caused by Ob-Rb gene silencing, which further confirmed that ASM inhibition blocked leptin resistance in RAECs.

Ceramide, the backbone of all sphingolipids, is generated in response to a variety of mediators, including proinflammatory cytokines, oxidative stress, and free fatty acids $[42,43]$. Obesity is associated with increased ceramide content in target tissues. For example, obesity affects the pathways of sphingolipid metabolism in adipose tissue [44]. On the other hand, the excess accumulation of ceramide contributes to the development of obesity and associated complications. In cultured adipocytes, ceramide directly induces SOCS3, suggesting a direct role for ceramide in the regulation of metabolism and energy expenditure [23]. Ceramide mediates obesity-related vascular dysfunction through the PP2A-mediated disruption of the eNOS/Akt/Hsp90 signaling complex [45]. In the current study, we found that ceramide significantly induced leptin resistance, as shown by the abnormal expression of SOCS3, Ob-Rb, and p-STAT3. For the first time, we demonstrated that ceramide has a direct role in leptin resistance, which also accounts for a key mechanism underlying the regulation of ceramide in obesity. In the current study, it was found that ceramide induced an increase in the expression of SOCS3, but after using SCH772984 to inhibit ERK1/2 activation, the expression of SOCS3 was still upregulated by a gradient concentration of ceramide, which suggested that the expression of SOCS3 induced by ceramide might be independent of ERK1/2 activation. It has also been reported that ceramide directly induces SOCS3 expression in cultured adipocytes [23]. Other studies have found that ceramide can induce a significant increase in interleukin- 6 (IL-6) expression and that IL- 6 can induce acute SOCS3 expression, which might be one of the potential mechanisms by which ceramide regulates the expression of SOCS3 [46, 47]. However, the detailed mechanisms still need further study.

Although Ob-R downregulation may be involved in the pathogenesis of leptin resistance, the molecular mechanisms of receptor downregulation remain unclear. It has been reported that the expression of Ob-Rb is positively correlated with ERK activation and that the administration of PD-98059, a specific mitogen-activated protein kinase kinase 1 inhibitor, can prevent the increase in Ob-Rb mRNA induced by leptin [20]. In the present study, we also found that elevated ceramide inhibited the expression of $\mathrm{Ob}-\mathrm{Rb}$ and the phosphorylation of STAT3 by suppressing ERK activation. Therefore, ERK mediated the regulation of the expression of Ob-Rb in RAECs by ceramide. However, further study is needed to determine how ERK regulates Ob-Rb expression.

Endothelial dysfunction is an increasingly recognized cause of cardiovascular disease and can be impaired during leptin resistance [48]. The assessment of endothelial function is primarily based on the release of NO and the activity of endothelial eNOS [49]. eNOS is the key enzyme that produces NO and participates in several biological processes, such as vasodilatation and platelet aggregation inhibition [50]. The current study found that ASM downregulation, compared with PA incubation or Ob-Rb siRNA transfected, increased the production of eNOS and NO in RAECs. It has been established that Akt deficiency directly leads to decreased eNOS activity and NO production [51]. Our previous study also demonstrated that the phosphorylation of Akt is regulated by the ASM/ceramide pathway [11]. Moreover, a defect in the regulation of hypothalamic Akt signaling by leptin may be one of the mechanisms of central leptin resistance [52]. The present study demonstrated that STAT3 and Akt interacted with each other and that the phosphorylation of Akt was regulated by STAT3 under leptin resistance. Thus, the data suggested that ASM downregulation improved the Ob-Rb/STAT3/Akt/eNOS signaling pathway to increase NO production in RAECs, providing additional evidence that ASM regulates endothelial leptin resistance.

Obesity represents a state of leptin resistance and is associated with elevated circulating leptin levels and HFD feeding-induced leptin resistance in rats $[53,54]$. In our in vivo study, we found that specific inhibitors of ASM inhibited serum leptin concentrations and reduced the weight gain of rats. This result is consistent with a previous study showing that HFD-induced body weight gain and plasma lipid and leptin concentrations in mice are significantly attenuated by AMI $[26,27]$. The decrease in the leptin concentration may be secondary to the improvement of leptin sensitivity. In addition, it has also been demonstrated that the inhibition of ASM can correct the abnormal metabolism that contributes to leptin resistance [55]. AMI and IMI are used as antidepressants, and in the brain, they affect neurotransmission and multiple pathways, including food intake and energy expenditure. Considering the broad role of these two drugs, our data suggested that the regulation of abnormal metabolism by $\mathrm{AMI}$ and IMI might be associated with ASM downregulation in high-fat diet-fed rats.

HFD feeding produces leptin resistance in rats and impaired endothelium-dependent relaxation in isolated aortas, providing a well-characterized model of endothelial dysfunction. Reduced NO is thought to aggravate endothelial dysfunction and the loss of vasodilation [56]. Leptin can induce vasodilatation through the Ob-Rb/STAT3/Akt signal pathways [57], and endothelial leptin 


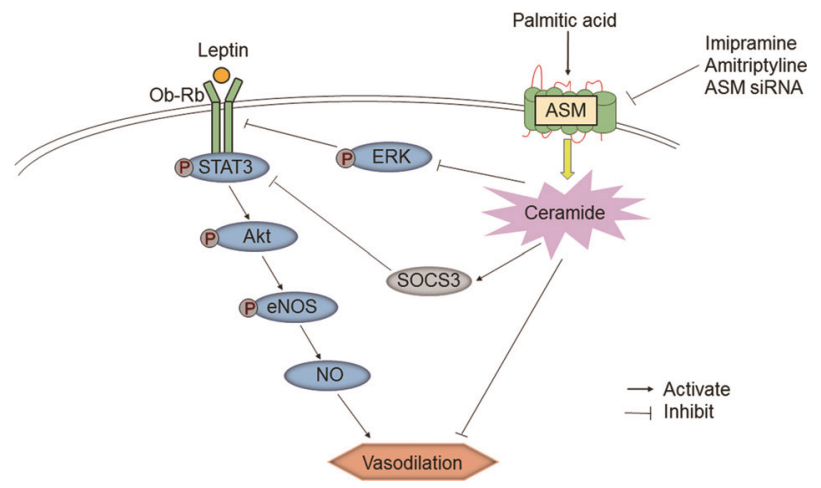

Fig. 8 Schematic diagram showing the effect of ASM downregulation on leptin resistance. ASM downregulation ameliorated endothelial leptin resistance and improved the Ob-Rb/STAT3/Akt/ eNOS signaling pathway to increase NO production, consequently resulting in the relief of vasodilatation dysfunction.

resistance impairs eNOS activation and reduces endotheliumdependent vasodilation [58]. Moreover, the ASM/ceramide pathway mediates vascular dysfunction by regulating the Akt/eNOS/ NO signaling pathway, and the inhibition of the ASM/ceramide pathway can improve endothelium-dependent vasodilation $[59,60]$. Here, the levels of serum eNOS and NO in HFD-fed rats were normalized by AMI and IMI. Moreover, ASM downregulation protected endothelium-dependent vasodilation in response to $\mathrm{ACh}$ and leptin. Furthermore, aortic rings preincubated with $L$-Arg or L-NNA indicated the effective role of ASM downregulation on the basal or inducible release of endothelial NO. Thus, the improvement of vascular function by ASM downregulation originated from the enhancement of eNOS/NO signal associated with the attenuation of leptin resistance. Consistent with the in vitro data, the immunohistochemistry and double immunofluorescence results suggested that ASM was activated in the thoracic aortic endothelium of HFD-fed rats. Moreover, the inhibition of ASM significantly decreased the level of SOCS3 and increased the level of Ob-Rb and p-STAT3, which further confirmed that ASM downregulation ameliorated progressive endothelial leptin resistance, consequently resulting in the relief of vasodilatation dysfunction. However, due to the regulation of abnormal metabolism and leptin by AMI and IMI, our data suggested that the vasodilatation dysfunction improvement originated from ASM downregulation in the aortic endothelium as well as the regulation of abnormal metabolism.

Taken together, our data demonstrated that ASM downregulation alleviated leptin resistance and consequently improved endothelial function, which was associated with a reduction in SOCS3 and an increase in the Ob-Rb/STAT3/Akt/eNOS/NO signaling pathway in endothelial cells (Fig. 8). These current findings revealed a novel mechanism of endothelial leptin resistance. ASM is regarded as a promising potential therapeutic target for cardiovascular disease associated with leptin resistance. In addition, the more specific approach of silencing ASM in rat endothelial cells using viral vector delivery is better than administering ASM inhibitors to regulate endothelial leptin resistance.

\section{ACKNOWLEDGEMENTS}

This work was supported by the National Natural Science Foundation of China (Nos. 81570413, 81773732, and 81973509).

\section{AUTHOR CONTRIBUTIONS}

$\mathrm{BBC}$ performed the experiments and wrote the original draft; YNL performed the experiments; $\mathrm{MX}$ designed the project and revised the manuscript.

\section{ADDITIONAL INFORMATION}

The online version of this article (https://doi.org/10.1038/s41401-019-0328-3) contains supplementary material, which is available to authorized users.

Competing interests: The authors declare no competing interests.

\section{REFERENCES}

1. Knight ZA, Hannan KS, Greenberg ML, Friedman JM. Hyperleptinemia is required for the development of leptin resistance. PLoS One. 2010;5:e11376.

2. Hubert A, Bochenek ML, Schutz E, Gogiraju R, Munzel T, Schafer K. Selective deletion of leptin signaling in endothelial cells enhances neointima formation and phenocopies the vascular effects of diet-induced obesity in mice. Arterioscler Thromb Vasc Biol. 2017;37:1683-97.

3. Myers MG, Cowley MA, Munzberg H. Mechanisms of leptin action and leptin resistance. Annu Rev Physiol. 2008;70:537-56.

4. Engin A. Diet-induced obesity and the mechanism of leptin resistance. Adv Exp Med Biol. 2017;960:381-97.

5. Jenkins RW, Canals D, Hannun YA. Roles and regulation of secretory and lysosomal acid sphingomyelinase. Cell Signal. 2009;21:836-46.

6. Smith EL, Schuchman EH. The unexpected role of acid sphingomyelinase in cell death and the pathophysiology of common diseases. FASEB J. 2008;22:3419-31.

7. Zeidan YH, Hannun YA. The acid sphingomyelinase/ceramide pathway: biomedical significance and mechanisms of regulation. Curr Mol Med. 2010;10:454-66.

8. Gulbins E, Walter S, Becker KA, Halmer R, Liu Y, Reichel M, et al. A central role for the acid sphingomyelinase/ceramide system in neurogenesis and major depression. J Neurochem. 2015;134:183-92.

9. Pan W, Yu J, Shi R, Yan L, Yang T, Li Y, et al. Elevation of ceramide and activation of secretory acid sphingomyelinase in patients with acute coronary syndromes. Coron Artery Dis. 2014;25:230-5.

10. Xu M, Xia M, Li XX, Han WQ, Boini KM, Zhang F, et al. Requirement of translocated lysosomal V1 $\mathrm{H}(+)$-ATPase for activation of membrane acid sphingomyelinase and raft clustering in coronary endothelial cells. Mol Biol Cell. 2012;23:1546-57.

11. Li X, Jin SJ, Su J, Li XX, Xu M. Acid sphingomyelinase down-regulation alleviates vascular endothelial insulin resistance in diabetic rats. Basic Clin Pharmacol Toxicol. 2018;123:645-59.

12. Cheng L, Yu Y, Szabo A, Wu Y, Wang H, Camer D, et al. Palmitic acid induces central leptin resistance and impairs hepatic glucose and lipid metabolism in male mice. J Nutr Biochem. 2015;26:541-8.

13. Palau VE, Chakraborty K, Wann D, Lightner J, Hilton K, Brannon $M$, et al. $Y$-Tocotrienol induces apoptosis in pancreatic cancer cells by upregulation of ceramide synthesis and modulation of sphingolipid transport. BMC Cancer. 2018;18:564.

14. Yuan XW, Han SF, Zhang JW, Xu JY, Qin LQ. Leucine supplementation improves leptin sensitivity in high-fat diet fed rats. Food Nutr Res. 2015;59:27373.

15. Herbet M, Gawronska-Grzywacz M, Izdebska M, Piatkowska-Chmiel I, JagielloWojtowicz E. Impact of combined treatment with rosuvastatin and antidepressants on liver and kidney function in rats. Exp Ther Med. 2016;11:1459-64.

16. Babenko NA, Garkavenko VV, Storozhenko GV, Timofiychuk OA. Role of acid sphingomyelinase in the age-dependent dysregulation of sphingolipids turnover in the tissues of rats. Gen Physiol Biophys. 2016;35:195-205.

17. Li N, Zhao Y, Yue Y, Chen L, Yao Z, Niu W. Liraglutide ameliorates palmitateinduced endothelial dysfunction through activating AMPK and reversing leptin resistance. Biochem Biophys Res Commun. 2016;478:46-52.

18. Santoro A, Mattace Raso G, Meli R. Drug targeting of leptin resistance. Life Sci. 2015;140:64-74.

19. Thon M, Hosoi T, Chea C, Ozawa K. Loss of stearoyl-coA desaturase-1 activity induced leptin resistance in neuronal cells. Biol Pharm Bull. 2017;40:1161-4.

20. Maroni P, Citterio L, Piccoletti R, Bendinelli P. Sam68 and ERKs regulate leptininduced expression of $\mathrm{Ob}-\mathrm{Rb}$ mRNA in $\mathrm{C} 2 \mathrm{C} 12$ myotubes. Mol Cell Endocrinol. 2009;309:26-31.

21. Simonis A, Hebling S, Gulbins E, Schneider-Schaulies S, Schubert-Unkmeir A. Differential activation of acid sphingomyelinase and ceramide release determines invasiveness of Neisseria meningitidis into brain endothelial cells. PLoS Pathog. 2014; 10:e1004160.

22. Zou H, Liu Y, Wei D, Wang T, Wang K, Huang $S$, et al. Leptin promotes proliferation and metastasis of human gallbladder cancer through OB-Rb leptin receptor. Int J Oncol. 2016;49:197-206.

23. Yang G, Badeanlou L, Bielawski J, Roberts AJ, Hannun YA, Samad F. Central role of ceramide biosynthesis in body weight regulation, energy metabolism, and the metabolic syndrome. Am J Physiol Endocrinol Metab. 2009;297:E211-24.

24. Siragusa M, Fleming I. The eNOS signalosome and its link to endothelial dysfunction. Pflug Arch. 2016;468:1125-37. 
25. Jin S, Teng X, Xiao L, Xue H, Guo Q, Duan X, et al. Hydrogen sulfide ameliorated LNAME-induced hypertensive heart disease by the Akt/eNOS/NO pathway. Exp Biol Med (Maywood). 2017;242:1831-41.

26. Boini KM, Zhang C, Xia M, Poklis JL, Li PL. Role of sphingolipid mediator ceramide in obesity and renal injury in mice fed a high-fat diet. J Pharmacol Exp Ther. 2010;334:839-46.

27. Fucho R, Martinez L, Baulies A, Torres S, Tarrats N, Fernandez A, et al. ASMase regulates autophagy and lysosomal membrane permeabilization and its inhibition prevents early stage non-alcoholic steatohepatitis. J Hepatol. 2014;61:1126-34.

28. Lembo G, Vecchione C, Fratta L, Marino G, Trimarco V, d'Amati G, et al. Leptin induces direct vasodilation through distinct endothelial mechanisms. Diabetes. 2000;49:293-7.

29. Arnalich-Montiel A, Gonzalez MC, Delgado-Baeza E, Delgado-Martos MJ, Condezo-Hoyos L, Martos-Rodriguez A, et al. Short-term esmolol improves coronary artery remodeling in spontaneously hypertensive rats through increased nitric oxide bioavailability and superoxide dismutase activity. Biomed Res Int. 2014;2014:531087.

30. Shetty GK, Matarese G, Magkos F, Moon HS, Liu X, Brennan AM, et al. Leptin administration to overweight and obese subjects for 6 months increases free leptin concentrations but does not alter circulating hormones of the thyroid and IGF axes during weight loss induced by a mild hypocaloric diet. Eur J Endocrinol. 2011;165:249-54.

31. Farr OM, Gavrieli A, Mantzoros CS. Leptin applications in 2015: what have we learned about leptin and obesity? Curr Opin Endocrinol Diabetes Obes. 2015;22:353-9.

32. Contreras C, Gonzalez-Garcia I, Martinez-Sanchez N, Seoane-Collazo P, Jacas J, Morgan DA, et al. Central ceramide-induced hypothalamic lipotoxicity and ER stress regulate energy balance. Cell Rep. 2014;9:366-77.

33. Sergi $D$, Morris AC, Kahn DE, McLean FH, Hay EA, Kubitz P, et al. Palmitic acid triggers inflammatory responses in N42 cultured hypothalamic cells partially via ceramide synthesis but not via TLR4. Nutr Neurosci. 2018;21:1-14.

34. Liu ZJ, Endoh A, Li R, Ohzeki T. Effects of leptin and dexamethasone on long and short leptin receptor mRNA. Pediatr Int. 2004;46:561-4.

35. Tena-Sempere M, Manna PR, Zhang FP, Pinilla L, Gonzalez LC, Dieguez C, et al. Molecular mechanisms of leptin action in adult rat testis: potential targets for leptin-induced inhibition of steroidogenesis and pattern of leptin receptor messenger ribonucleic acid expression. J Endocrinol. 2001;170:413-23.

36. Jin X, Fukuda N, Su J, Takagi H, Lai Y, Lin Z, et al. Effects of leptin on endothelial function with $\mathrm{OB}-\mathrm{Rb}$ gene transfer in Zucker fatty rats. Atherosclerosis. 2003;169:225-33.

37. Wang MY, Koyama K, Shimabukuro M, Newgard CB, Unger RH. OB-Rb gene transfer to leptin-resistant islets reverses diabetogenic phenotype. Proc Natl Acad Sci U S A. 1998;95:714-8

38. Lopez M, Seoane LM, Tovar S, Garcia MC, Nogueiras R, Dieguez C, et al. A possible role of neuropeptide $Y$, agouti-related protein and leptin receptor isoforms in hypothalamic programming by perinatal feeding in the rat. Diabetologia. 2005;48:140-8.

39. Wang B, Chandrasekera PC, Pippin JJ. Leptin- and leptin receptor-deficient rodent models: relevance for human type 2 diabetes. Curr Diabetes Rev. 2014;10:131-45.

40. Shimizu Y, Son C, Aotani D, Nomura H, Hikida T, Hosoda K, et al. Role of leptin in conditioned place preference to high-fat diet in leptin-deficient $o b / o b$ mice. Neurosci Lett. 2017;640:60-3.

41. Zhang Y, Proenca R, Maffei M, Barone M, Leopold L, Friedman JM. Positional cloning of the mouse obese gene and its human homologue. Nature. 1994;372:425-32.
42. Jazvinscak Jembrek M, Hof PR, Simic G. Ceramides in Alzheimer's disease: key mediators of neuronal apoptosis induced by oxidative stress and abeta accumulation. Oxid Med Cell Longev. 2015;2015:346783.

43. Zabielski P, Blachnio-Zabielska AU, Wojcik B, Chabowski A, Gorski J. Effect of plasma free fatty acid supply on the rate of ceramide synthesis in different muscle types in the rat. PLoS One. 2017;12:e0187136.

44. Blachnio-Zabielska AU, Pulka M, Baranowski M, Nikolajuk A, Zabielski P, Gorska M, et al. Ceramide metabolism is affected by obesity and diabetes in human adipose tissue. J Cell Physiol. 2012;227:550-7.

45. Zhang QJ, Holland WL, Wilson L, Tanner JM, Kearns D, Cahoon JM, et al. Ceramide mediates vascular dysfunction in diet-induced obesity by PP2A-mediated dephosphorylation of the eNOS-Akt complex. Diabetes. 2012;61:1848-59.

46. Laulederkind SJ, Bielawska A, Raghow R, Hannun YA, Ballou LR. Ceramide induces interleukin 6 gene expression in human fibroblasts. J Exp Med. 1995;182:599-604.

47. Gao S, Durstine JL, Koh HJ. Acute myotube protein synthesis regulation by IL-6related cytokines. Am J Physiol Cell Physiol. 2017;313:C487-c500.

48. Yang R, Sikka G, Larson J, Watts VL, Niu X, Ellis CL, et al. Restoring leptin signaling reduces hyperlipidemia and improves vascular stiffness induced by chronic intermittent hypoxia. Am J Physiol Heart Circ Physiol. 2011;300:H1467-76.

49. McNamara TC, Keen JT, Simmons GH, Alexander LM, Wong BJ. Endothelial nitric oxide synthase mediates the nitric oxide component of reflex cutaneous vasodilatation during dynamic exercise in humans. J Physiol. 2014;592:5317-26.

50. Gkaliagkousi E, Ferro A. Nitric oxide signalling in the regulation of cardiovascular and platelet function. Front Biosci (Landmark Ed). 2011;16:1873-97.

51. Walther S, Pluteanu F, Renz S, Nikonova Y, Maxwell JT, Yang LZ, et al. Urocortin 2 stimulates nitric oxide production in ventricular myocytes via Akt- and PKAmediated phosphorylation of eNOS at serine 1177. Am J Physiol Heart Circ Physiol. 2014;307:H689-700.

52. Sahu M, Anamthathmakula P, Sahu A. Phosphodiesterase-3B-CAMP pathway of leptin signalling in the hypothalamus is impaired during the development of diet-induced obesity in FVB/N mice. J Neuroendocrinol. 2015;27:293-302.

53. Munzberg $\mathrm{H}$. Leptin-signaling pathways and leptin resistance. Forum Nutr. 2010;63:123-32.

54. Sainz N, Barrenetxe J, Moreno-Aliaga MJ, Martinez JA. Leptin resistance and dietinduced obesity: central and peripheral actions of leptin. Metabolism. 2015;64:35-46.

55. Sanguesa G, Roglans N, Montanes JC, Baena M, Velazquez AM, Sanchez RM, et al. Chronic liquid fructose, but not glucose, supplementation selectively induces visceral adipose tissue leptin resistance and hypertrophy in female Sprague-Dawley rats. Mol Nutr Food Res. 2018;62:e1800777.

56. Yin DD, Wang QC, Zhou X, Li Y. Endothelial dysfunction in renal arcuate arteries of obese Zucker rats: The roles of nitric oxide, endothelium-derived hyperpolarizing factors, and calcium-activated $\mathrm{K}^{+}$channels. PLoS One. 2017;12:e0183124.

57. Tune JD, Considine RV. Effects of leptin on cardiovascular physiology. J Am Soc Hypertens. 2007;1:231-41.

58. Daiber A, Xia N, Steven S, Oelze M, Hanf A, Kroller-Schon S, et al. New therapeutic implications of endothelial nitric oxide synthase (eNOS) function/dysfunction in cardiovascular disease. Int J Mol Sci. 2019;20: pii: E187. https://doi.org/10.3390/ ijms20010187.

59. Jiang M, Huang S, Duan W, Liu Q, Lei M. Inhibition of acid sphingomyelinase activity ameliorates endothelial dysfunction in $d b / d b$ mice. Biosci Rep. 2019;39: BSR20182144.

60. Wang A, Li C, Liao J, Dong M, Xiao Z, Lei M. Ceramide mediates inhibition of the Akt/eNOS pathway by high levels of glucose in human vascular endothelial cells. J Pediatr Endocrinol Metab. 2013;26:31-8. 